\title{
RELATIVE GRÖBNER-SHIRSHOV BASES FOR ALGEBRAS AND GROUPS
}

\author{
L. A. BOKUT AND K. P. SHUM
}

\begin{abstract}
The notion of a relative Gröbner-Shirshov basis for algebras and groups is introduced. The relative composition lemma and relative (composition-)diamond lemma are established. In particular, it is shown that the relative normal forms of certain groups arising from Malcev's embedding problem are the irreducible normal forms of these groups with respect to their relative Gröbner-Shirshov bases. Other examples of such groups are given by showing that any group $G$ in a Tits system $(G, B, N, S)$ has a relative $(B$-) Gröbner-Shirshov basis such that the irreducible words are the Bruhat words of $G$.
\end{abstract}

\section{$\S 1$. INTRODUCTION}

In this paper we define the notion of a relative Gröbner-Shirshov basis and prove the relative Shirshov lemma and a relative composition-diamond lemma for $\Gamma$-algebras $(\Gamma$ semigroups, $\Gamma$-groups) presented by generators and defining relations. Here $\Gamma$ is a fixed subgroup of the algebra (semigroup, group).

As illustrations, in subsequent sections we consider a number of examples of $\Gamma$ semigroups and $\Gamma$-groups for a nontrivial $\Gamma$, calculate relative Gröbner-Shirshov bases for them, and indicate some applications. Here are these examples.

1) Let $Q_{4}$ (see [4]) be the following semigroup:

$$
\begin{aligned}
& Q_{4}=\operatorname{sgr}\left\langle a_{i}, b_{i}, c_{i}, s_{i}, t_{i}, 1 \leq i \leq 4, v_{0}, v_{1}\right| \\
& \left.\quad a_{i} s_{i}=c_{i} v_{0}, b_{i} s_{i+1}=c_{i} v_{1}, b_{i} t_{i+1}=a_{i} t_{i}, 1 \leq i \leq 4\right\rangle,
\end{aligned}
$$

where $s_{5}=s_{1}, t_{5}=t_{1}$.

Let $R$ be the algebra of formal power series over $Q_{4}$ with coefficients in $G F(2)$. In other words, the algebra $R$ is the completion of the semigroup algebra $G F(2)\left(Q_{4}\right)$ relative to the norm $2^{-\operatorname{deg}(f)}$, where $\operatorname{deg}(f)$ is the natural degree of an element $f \in G F(2)\left(Q_{4}\right)$. We fix the presentation of the multiplicative semigroup $R^{*}$ of $R$ that was found in [5]. Then $R^{*}$ is a $\Gamma$-semigroup, where $\Gamma$ is the group of units of $R$ ( $\Gamma$ consists of series with nonzero constant terms).

By definition, to be a $\Gamma$-semigroup means that for any element $q$ among the selected generators for $R^{*}$ there are two isomorphic subgroups $\Gamma_{q}$ and $\Gamma_{q}^{\prime}$ of $\Gamma$ with

$$
\gamma q=q \gamma^{\prime},
$$

where $\gamma \in \Gamma_{q}$ and $\gamma^{\prime} \in \Gamma_{q}^{\prime}$. In our case,

$$
\Gamma_{q}=\{1+q A \mid A \in R\}, \quad \Gamma_{q}^{\prime}=\{1+A q \mid A \in R\} .
$$

2000 Mathematics Subject Classification. Primary 16S15, 16S34, 20C05, $20 \mathrm{C} 07$.

Key words and phrases. Relative Gröbner-Shirshov bases, irreducible normal form, rewriting system, Tits systems, Malcev's problem.

Supported by RFBR and SS, grant no. 344.2008.1, and by UGC (HK), grant no. 2060187, 2002/04. 
It should be noted that the above $\Gamma$-semigroup structure of $R^{*}$ was absolutely crucial in proving that $R^{*}$ is embeddable into a group (see [6, 8, 9]), though $R$ is not embeddable into any skew field (see $\S 3$ ). The relative composition-diamond lemma allows us to say that the main technical result of [6, 8, 9] consists in finding a relative Gröbner-Shirshov basis of the universal group $G\left(R^{*}\right)$ in an explicit form (see $\S 3$ ).

Recall that a universal group $G(P)$ of some semigroup presentation $P$ is the group with the same generators and defining relations as $P$.

2) Let $Q$ be any SNA-semigroup presentation in the sense of $[8$. This is a semigroup with defining relations of the form

$$
w h=u f,
$$

where $w, h, u, f$ are generators with some additional properties. One of the crucial properties of $Q$ is that $Q$ has no left (right) cycles of order not exceeding 3 in the sense of Adyan [1; in particular, no relations of the form

$$
w h=u f, \quad w_{1} h_{1}=u f_{1}, \quad w_{1} h_{2}=w f_{2}
$$

may be fulfilled in $Q$ simultaneously. This is not the case for the above semigroup $Q_{4}$. However, obviously, there are many examples of SNA-semigroups (cf. [8]).

Let $k$ be a field, and let $\overline{k Q}$ be the algebra of formal power series over $Q$ with coefficients in $k$. Again, the semigroup $\overline{k Q}^{*}$ is a $\Gamma$-semigroup, where $\Gamma$ is the group of units of $\overline{k Q}$. The universal group $G\left(\overline{k Q}^{*}\right)$ is also a $\Gamma$-group. Again, the relative composition-diamond lemma makes it possible to state the main technical result of [8] about SNA-semigroups as follows: a relative Gröbner-Shirshov basis of the universal group $G\left(\overline{k Q}^{*}\right)$ can be found explicitly (see $\S 3$ ).

As a result, any semigroup $\overline{k Q}^{*}$ is embeddable into a group (see [8]).

3 ) Let $(G, B, N, S)$ be a Tits system (see, e.g., 2]). Then $G$ can be viewed as a $\Gamma$ group for $\Gamma=B$ (see $\S 4$ ). From this point of view, the Bruhat normal form for $G$ is the irreducible normal form for $G$ with respect to a relative Gröbner-Shirshov basis of $G$ (see $\S 5$ ).

It should be noted that in the papers [4, 5, 6, 8, 9] cited above (see also 12]) the main goal was to solve the following Malcev embedding problem (cf. 23]) in the class of semigroup algebras:

Does there exist a semigroup algebra that is not embeddable into any skew field, but with the multiplicative semigroup embeddable into a group?

In $\S 4$ we discuss an analog of the Malcev problem for group algebras, as well as some other problems for them.

\section{§2. Relative composition lemma}

Let $X=\left\{x_{i}, i \in I\right\}$ be a well-ordered set (we transfer this order to $I$, that is, $x_{i}<x_{j}$ if $i<j$ ), and let $\Gamma$ be a group, the elements of which will be denoted by small greek letters $\gamma, \delta, \ldots$ with indices. Consider the multiplication table

$$
\gamma \delta=\mu
$$

for $\Gamma$.

Suppose that for any $x \in X$ two isomorphic subgroups $\Gamma_{x}$ and $\Gamma_{x}^{\prime}$ of $\Gamma$ are fixed, together with an isomorphism

$$
\partial_{x}: \Gamma_{x} \rightarrow \Gamma_{x}^{\prime} .
$$

Then we consider $k\langle X ; \Gamma\rangle$, the $\Gamma$-free algebra over a field $k$ and the set $X$ (see [14). In fact, this is an associative algebra with 1 , generated by $X$ and $\Gamma$, with the defining 
relations (1) and

$$
\gamma x=x \gamma^{\prime},
$$

where $x \in X, \gamma \in \Gamma_{x}, \gamma^{\prime}=\partial_{x}(\gamma) \in \Gamma_{x}^{\prime}$. If $\Gamma=1$, then this algebra is simply the free algebra over $k$ and $X$.

By definition, the $\Gamma$-words have the form

$$
u=\gamma_{0} x_{1} \cdots x_{k} \gamma_{k},
$$

where $k \geq 0, \gamma_{j} \in \Gamma, x_{i_{j}} \in X$. We denote the projection of $u$ to $X$ by $[u]$, that is,

$$
[u]=x_{i_{1}} x_{i_{2}} \cdots x_{i_{k}} .
$$

Also, we define

$$
|u|=|[u]|=k ;
$$

this is the length or the degree of $u$.

It is easily seen that two $\Gamma$-words $u=\gamma_{0} x_{i_{1}} \cdots x_{i_{k}} \gamma_{k}$ and $v=\delta_{0} x_{j_{1}} \delta_{1} \cdots x_{j_{l}} \delta_{l}$ are equal in the $\Gamma$-free algebra $k\langle X ; \Gamma\rangle$ if and only if

$$
k=l, \quad x_{i_{s}}=x_{j_{s}}, \quad 1 \leq s \leq k,
$$

and

$$
\gamma_{0}=\delta_{0} \gamma_{x_{i_{1}}}, \quad \gamma_{x_{i_{1}}}^{\prime} \gamma_{1}=\delta_{1} \gamma_{x_{i_{2}}}, \ldots, \gamma_{x_{i_{k}}}^{\prime} \gamma_{k}=\delta_{k}
$$

If $u=u_{1} v u_{2}$ for some $\Gamma$-words, then $v$ is called a $\Gamma$-subword of $u$. The relation $u v=w t$ implies immediately that $u=w a$ and $t=a v$, or $w=u a$ and $v=a t$, for some $\Gamma$-word $a$.

We fix a monomial order of $X$-words (5), such that $u<v \Rightarrow a u b<a v b$ for any $X$-words $u, v, a, b$, assuming that this order agrees with the order of $X$ (clearly, this is possible). Then, it gives rise to a quasi-order of $\Gamma$-words:

$$
u \preceq v \quad \text { if } \quad[u] \leq[v] .
$$

Thus,

$$
u \preceq v \Rightarrow a u b \preceq a v b
$$

for any $\Gamma$-words $u, v, a, b$.

Let $f \in k\langle X ; \Gamma\rangle$. We assume that $f$ is presented in the form

$$
f=\sum \alpha_{i} u_{i}
$$

where $\alpha_{i} \in k$ and the $u_{i}$ are pairwise distinct $\Gamma$-words.

For the above polynomial $f$, we use $\bar{f}$ to denote a leading monomial of $f$. In general, there are several leading monomials of $f$. In other words, $\bar{f}$ is a maximal $\Gamma$-word occurring in $f$ with a nonzero coefficient. If $\bar{f}$ is unique, then we call $f$ a strong polynomial and $\bar{f}$ the strong leading monomial of $f$.

We say that $f$ is monic strong if $f$ is strong with leading coefficient 1 (i.e., the coefficient of $\bar{f}$ is 1$)$.

Let $S \subset k\langle X ; \Gamma\rangle$. Then we call $S$ a monic strong set if any $s \in S$ is a monic strong polynomial. From now on, we use $S$ to denote some set of monic strong polynomials.

The composition of two monic strong polynomials $f, g$ can be defined, in essence, in the same way as in the case where $\Gamma=1$ (see, e.g., [15). Namely, we define

$$
(f, g)_{w}= \begin{cases}f v-u g & \text { if } \quad w=\bar{f} v=u \bar{g},|\bar{f}|>|u|, \\ f-u g v & \text { if } \quad w=\bar{f}=u \bar{g} v\end{cases}
$$

where $w, v, u$ are $\Gamma$-words. In the second case of (8), the transformation

$$
f \mapsto f-u g v
$$


is called elimination of the leading word (briefly, ELW) of $g$ in $f$. In both cases of (8), for any leading word $\overline{(f, g)_{w}}$ we have

$$
\overline{(f, g)_{w}}<w
$$

though $(f, g)_{w}$ may fail to be a strong polynomial.

Let $f$ be a polynomial (not necessarily strong) such that a leading monomial $\bar{f}$ of $f$ is of the form

$$
\bar{f}=u \bar{g} v,
$$

where $g$ is a monic strong polynomial. Suppose that $f$ is $\bar{f}$-monic, that is, $\bar{f}$ occurs in $f$ with coefficient 1 . Then the transformation (9) (elimination of the leading word of $g$ in $f$ ) is applicable. In this case, for the polynomial $f-u g v$ the number of occurrences of leading words that are quasi-equal to $\bar{f}$ is smaller than that for $f$. By using this crucial observation, we obtain the following important property: any sequence of ELW's of strong monic polynomials is finite; i.e., any sequence

$$
f_{1} \rightarrow f_{2} \rightarrow \cdots \rightarrow f_{n} \rightarrow \cdots
$$

is finite, where the leading word of a strong monic polynomial is eliminated at each step.

A composition of the form (8) is said to be trivial relative to $S$ (more precisely, relative to $S$ and $w)$, and we write $(f, g)_{w} \equiv \bmod (S, w)$ if

$$
(f, g)_{w}=\sum \alpha_{i} u_{i} s_{i} v_{i}
$$

where $\alpha_{i} \in k, s \in S, u_{i}, v_{i}$ are $\Gamma$-words, and

$$
u_{i} \bar{s}_{i} v_{i} \prec w .
$$

In particular, if $(f, g)_{w}$ goes to zero under the action of ELW's of $S$, then $(f, g)_{w}$ is trivial relative to $S, w$ (see the proof of the "if" part in the next theorem).

A monic strong set $S$ is called a relative $\Gamma$-Gröbner-Shirshov basis (in $k\langle X ; \Gamma\rangle$ ) if any composition $(f, g)_{w}$ of elements $f, g$ of $S$ is trivial $\bmod (S, w)$.

Remark 2.1. For the first time, condition (11) (for $\Gamma=1$ ) was stated in [10] for the case of Lie algebras; then it was used in [11] for associative algebras, and also in 22 . for commutative algebras. In fact, condition (11) is easier to check, as compared to a stronger condition involving ELW's, though the latter condition is algorithmic (for a recursive $S$ ). For the definition of a (relative) Gröbner-Shirshov basis, any of these two conditions can be used (see below).

With the above concepts at hand, now we can formulate the relative composition lemma. This is an analog (even a generalization) of Shirshov's composition lemma 25] (see, e.g., [15). In what follows, $\operatorname{id}(S)$ stands for the two-sided ideal of the algebra $k\langle X ; \Gamma\rangle$ generated by $S$.

Theorem 2.1 (Relative composition lemma). Let $S \subset k\langle X ; \Gamma\rangle$ be a strong monic set of the $\Gamma$-free algebra $k\langle X ; \Gamma\rangle$. Then $S$ is a relative Gröbner-Shirshov basis if and only if for any $f \in \operatorname{id}(S)$ we have $\bar{f}=a \bar{s} b$ for some leading monomial $\bar{f}$ of $f$ and $s \in S$.

Proof. The "only if" part. Let $S$ be a relative Gröbner-Shirshov basis, and let $f \in \operatorname{id}(S)$. Then

$$
f=\sum_{i=1}^{n} \alpha_{i} \alpha_{i} s_{i} b_{i}
$$

where $\alpha_{i} \in k$, the $\alpha_{i}, b_{i}$ are $\Gamma$-words, and $s_{i} \in S$. It is easily seen that each $a_{i} s_{i} b_{i}$ is a monic strong polynomial with the leading word $a_{i} \bar{s}_{i} b_{i}$. Now, we arrange these leading 
words in nonincreasing order by

$$
w=a_{1} \bar{s}_{1} b_{1}=a_{2} \bar{s}_{2} b_{2}=\cdots=a_{k} \bar{s}_{k} \succeq a_{k+1} \bar{s}_{k+1} b_{k+1} \succeq \cdots \succeq a_{n} \bar{s}_{n} b_{n}
$$

and $w \neq a_{k+i} s_{k+i} b_{k+i}, i \geq 1$. If $k=1$, then on the right-hand side of (13) there are no $\Gamma$-words equal to $a_{1} \bar{s}_{1} b_{1}$. This shows that $\bar{f}=a_{1} \bar{s}_{1} b_{1}$ is a leading word of $f$. Let $k \geq 2$, and let

$$
a_{1} \bar{s}_{1} b_{1}=a_{2} \bar{s}_{2} b_{2}, \quad\left[a_{1}\right]\left[\bar{s}_{1}\right]\left[b_{1}\right]=\left[a_{2}\right]\left[\bar{s}_{2}\right]\left[b_{2}\right] .
$$

The following three cases arise.

1) The subwords $\left[\bar{s}_{1}\right]$ and $\left[\bar{s}_{2}\right]$ do not intersect. For example, let

$$
\left[a_{2}\right]=\left[a_{1}\right]\left[\bar{s}_{1}\right][d], \quad\left[b_{1}\right]=[d]\left[\bar{s}_{2}\right]\left[b_{2}\right],
$$

where $[d]$ is an $X$-word. Then (14) implies that

$$
a_{2}=a_{1} \bar{s}_{1} d, \quad b_{1}=d \bar{s}_{2} b_{2}
$$

for some $\Gamma$-word $d$.

Consequently,

$$
\begin{aligned}
\alpha_{1} a_{1} \bar{s}_{1} b_{1} & +\alpha_{2} a_{2} s_{2} b_{2}=\left(\alpha_{1}+\alpha_{2}\right) a_{1} s_{1} b_{1}-\alpha_{2}\left(a_{1} s_{1} b_{1}-a_{2} s_{2} b_{2}\right) \\
& =\left(\alpha_{1}+\alpha_{2}\right) a_{1} s_{1} b_{1}-\alpha_{2}\left(a_{1} s_{1} d \bar{s}_{2} b_{2}-a_{1} \bar{s}_{1} d s_{2} b_{2}\right) \\
& \left.=\left(\alpha_{1}+\alpha_{2}\right) a_{1} s_{1} b_{1}-\alpha_{2}\left(a_{1} s_{1} d\left(\bar{s}_{2}-s_{2}\right) b_{2}+a_{1}\left(s_{1}-\bar{s}_{1}\right)\right) d s_{2} b_{2}\right),
\end{aligned}
$$

where

and

$$
a_{1} s_{1} d\left(\bar{s}_{2}-s_{2}\right) b_{2}=\sum \gamma_{j} c_{j} s_{j} d_{j}, \quad c_{j} \bar{s}_{j} d_{j} \prec w,
$$

$$
a_{1}\left(s_{1}-\bar{s}_{1}\right) d s_{2} b_{2}=\sum \delta_{j} c_{j}^{\prime} s_{j} d_{j}^{\prime}, \quad c_{j}^{\prime} \bar{s}_{j} d_{j}^{\prime} \prec w .
$$

The above identities allow us to rewrite (13) with a smaller $k$.

2) The subwords $\left[\bar{s}_{1}\right]$ and $\left[\bar{s}_{2}\right]$ in (14) have nonempty intersection, but none of them includes the other. For example, let

$$
\left[\bar{s}_{1}\right]=[a][c], \quad\left[\bar{s}_{2}\right]=[c][b], \quad[c] \neq 1 .
$$

Then (14) implies that

$$
a_{2}=a_{1} a, \quad b_{1}=b b_{2}, \quad \bar{s}_{1} b=a \bar{s}_{2}=w_{1}, \quad\left|\bar{s}_{1}\right|>|a|,
$$

where $a, b$ are some $\Gamma$-words. Indeed,

$$
\bar{s}_{1}=a^{\prime} c^{\prime}, \quad \bar{s}_{2}=c^{\prime} b^{\prime}, \quad a_{1} a^{\prime} c^{\prime} b_{1}=a_{2} c^{\prime} b^{\prime} b_{2}
$$

for some $\Gamma$-words $a^{\prime}, c^{\prime}, b^{\prime}$. Then

$$
a_{2}=a_{1} a^{\prime} \gamma, \quad \gamma c^{\prime}=c^{\prime} \delta, \quad b_{1}=\delta b^{\prime} b_{2}
$$

for some $\gamma, \delta$ in $\Gamma$. It remains to put $a=a^{\prime} \gamma, b=\delta b^{\prime}$.

Now we can deduce the formula

$$
\begin{aligned}
\alpha_{1} a_{1} s_{1} b_{1}+\alpha_{2} a_{2} s_{2} b_{2} & =\left(\alpha_{1}+\alpha_{2}\right) a_{1} s_{1} b_{1}-\alpha_{2}\left(a_{1} s_{1} b_{1}-a_{2} s_{2} b_{2}\right) \\
& =\left(\alpha_{1}+\alpha_{2}\right) a_{1} s_{1} b_{1}-\alpha_{2}\left(a_{1} s_{1} b b_{2}-a_{1} a s_{2} b_{2}\right) \\
& =\left(\alpha+\alpha_{2}\right) a_{1} s_{1} b_{1}-\alpha_{2} a_{1}\left(s_{1} b-a s_{2}\right) b_{2} \\
& =\left(\alpha_{1}+\alpha_{2}\right) a_{1} s_{1} b_{1}-\alpha_{2} a_{1}\left(s_{1}, s_{2}\right)_{w_{1}} b_{2} .
\end{aligned}
$$

Since $S$ is a Gröbner-Shirshov basis, the composition $\left(s_{1}, s_{2}\right) w_{1}$ is trivial $\bmod \left(S, w_{1}\right)$, and we obtain

where $s_{j} \in S$ and

$$
a_{1}\left(s_{1}, s_{2}\right)_{w_{1}} b_{2}=\sum \gamma_{j} a_{1} c_{j} s_{j} d_{j} b_{2}
$$

$$
c_{j} \bar{s}_{j} d_{j} \prec w_{1}
$$


Then

$$
a_{1} c_{j} \bar{s}_{j} d_{j} b_{2} \prec a_{1} w_{1} b_{2}=w .
$$

By using the above relations, we can rewrite (13) with a smaller $k$.

3) One of the subwords $\left[\bar{s}_{1}\right],\left[\bar{s}_{2}\right]$ is a subword of the other.

For example, let

$$
\left[\bar{s}_{1}\right]=[a]\left[\bar{s}_{2}\right][b] .
$$

Arguing as in the preceding case, we deduce from (14) that

$$
a_{2}=a_{1} a^{\prime} \gamma, \quad \gamma \bar{s}_{2}=\bar{s}_{2} \delta, \quad \delta b_{2}=b^{\prime} b_{1}
$$

for some $\Gamma$-words $a^{\prime}, b^{\prime}$ and some elements $\gamma, \delta \in \Gamma$. Putting $a=a^{\prime} \gamma, b=\delta^{-1} b^{\prime}$, we see that

$$
w_{1}=\bar{s}_{1}=a \bar{s}_{2} b, \quad a_{2}=a_{1} a, \quad b_{2}=b b_{1}
$$

for some $\Gamma$-words $a, b$. Now, we can deduce the formula

$$
\begin{aligned}
\alpha_{1} a_{1} s_{1} b_{1}+\alpha_{2} a_{2} s_{2} b_{2} & =\left(\alpha_{1}+\alpha_{2}\right) a_{1} s_{1} b_{1}-\alpha_{2}\left(a_{1} s_{1} b_{1}-a_{2} s_{2} b_{2}\right) \\
& =\left(\alpha_{1}+\alpha_{2}\right) a_{1} s_{1} b_{1}-\alpha_{2}\left(a_{1} s_{1} b_{1}-a_{1} a s_{2} b b_{1}\right) \\
& =\left(\alpha_{1}+\alpha_{2}\right) a_{1} s_{1} b_{1}-\alpha_{2} a_{1}\left(s_{1}-a s_{2} b\right) b_{1} \\
& =\left(\alpha_{1}+\alpha_{2}\right) a_{1} s_{1} b_{1}-\alpha_{2} a_{2}\left(s_{1}, s_{2}\right)_{w_{1}} b_{1} .
\end{aligned}
$$

As before,

$$
a_{1}\left(s_{1}, s_{2}\right)_{w_{1}} b_{1}=\sum \gamma_{j} a_{1} c_{j} s_{j} d_{j} b_{1},
$$

where $s_{j} \in S$ and

$$
c_{j} \bar{s}_{j} d_{j} \succeq w_{1}, \quad a_{1} c_{j} \bar{s}_{j} d_{j} b_{1} \prec a_{1} w_{1} b_{1}=w .
$$

After this, we can use the above relations to rewrite (13) with a smaller $k$.

Thus, by induction on $k$, we can easily complete the proof of the "only if" part.

The "if" part. First, we let $(f, g)_{w}$ be a composition of elements of $S$ relative to $w$. Then

$$
(f, g)_{w}=\sum \beta_{j} u_{j}, \quad u_{j} \prec w .
$$

By using ELW's of $S$ in $(f, g)_{w}$, we can present $(f, g)_{w}$ in a form $h$ such that any leading monomial $\bar{h}$ of $h$ contains no subwords $\bar{s}, s \in S$. Clearly, we have $h=0$, because $h \in \operatorname{id}(S)$. By the construction of $h$,

$$
(f, g)_{w}=\sum \alpha_{i} a_{i} s_{i} b_{i}, \quad s_{i} \in S, \quad a_{i} \bar{s}_{i} b_{i} \prec w,
$$

i.e., $(f, g)_{w}=0(\bmod (S, w))$, and precisely this is required for $S$ to be a relative GröbnerShirshov basis. To clarify the last formula, let $u_{j}$ be a leading monomial in the initial presentation of $(f, g)_{w}, u_{j} \prec w$. We know that $u_{j}<w$. Suppose that $u_{j}=a \bar{s} b$ for some $s \in S$. Then

$$
(f, g)_{w}-\beta_{j} a s b
$$

has a smaller number of leading monomials than $(f, g)_{w}$, and $a \bar{s} b \prec w$. After a finite number of such steps, we can obtain the zero polynomial and, with it, the required presentation of $(f, g)_{w}$.

Theorem 2.2 implies the following useful proposition. This is an analog (again, a generalization) of the composition-diamond lemma (see, e.g., [15]). We say that a $\Gamma$ word $u$ is irreducible relative to some $S$ (Irr-word for short) if $u \neq a \bar{s} b, s \in S$. The set of all Irr-words relative to $S$ is denoted by $\operatorname{Irr}(S)$ (this set was denoted by $P B W(S)$ in [15]). 
Theorem 2.2 (Relative composition-diamond lemma). Let $S \subset k\langle X ; \Gamma\rangle$ be a monic strong set. Then $S$ forms a relative Gröbner-Shirshov basis in $k\langle X ; \Gamma\rangle$ if and only if $\operatorname{Irr}(S)$ is a basis over $k$ of the algebra $k\langle X ; \Gamma \mid S\rangle$, that is, the $\Gamma$-algebra with generators $X$ and defining relations $S$.

Proof. The "only if" part. Using ELW's of $S$, we can easily check that every $f \in k\langle X ; \Gamma\rangle$ can be presented modulo id $(S)$ as a linear combination of Irr-words. On the other hand, all the Irr-words are linearly independent $\bmod \operatorname{id}(S)$.

The "if" part. Suppose that a composition $(f, g)_{w}$, where $f, g \in S$, is nontrivial. Then we can use ELW's of $S$ to present $(f, g)_{w}$ as a nontrivial linear combination of Irr-words plus some element of $\operatorname{id}(S)$. This contradicts the linear independence of the Irr-words.

The following corollary also characterizes the relative Gröbner-Shirshov bases in $k\langle X ; \Gamma\rangle$. This statement is well known for the case where $\Gamma=1$ (it dates back to [25] and [10]).

Corollary 2.3. Let $S \subset k\langle X ; \Gamma\rangle$ be a monic strong subset of $k\langle X ; \Gamma\rangle$. Then $S$ is a relative Gröbner-Shirshov basis in $k\langle X ; \Gamma\rangle$ if and only if any composition of elements of $S$ can be reduced to zero by ELW's of $S$.

Proof. The "only if" part. By using ELW's of $S$, we can represent a composition $(f, g)_{w}$, $f, g \in S$, as a linear combination of Irr-words. By the relative composition lemma, this linear combination must be trivial.

The "if" part. This part is clear: see the text after (12) and the proof of the "if" part of Theorem 2.3.

Recall that

$$
k\langle X ; \Gamma \mid S\rangle=k\langle X ; \Gamma\rangle / \operatorname{id}(S)
$$

is the $\Gamma$-algebra generated by $X$ with the set of defining relations $S$. We define the $\Gamma$-semigroups and $\Gamma$-groups in a similar way.

Let $X=\left\{x_{i}, i \in I\right\}, \Gamma, \Gamma_{x}, \Gamma_{x}^{\prime}, x \in X$, be as above. By a $\Gamma$-free semigroup $\operatorname{sgr}\langle X ; \Gamma\rangle$ we mean a semigroup with 1 that is generated by $X$ and $\Gamma$, with the defining relations (1) and (3). The elements of $\operatorname{sgr}\langle X ; \Gamma\rangle$ are the $\Gamma$-words (4) with the equality relation (6).

Let $S=\left\{\left(u_{j}, v_{j}\right), j \in J\right\}$ be a set of pairs of $\Gamma$-words. As usual, we introduce the $\Gamma$-semigroup with defining relations $S$ by

$$
P=\operatorname{sgr}\left\langle X ; \Gamma \mid u_{j}=v_{j}, j \in J\right\rangle=\operatorname{sgr}\langle X ; \Gamma \mid S\rangle
$$

(we identify the relation $u=v$ with the pair $(u, v)$ ). Then the semigroup algebra $k(P)$ is the $\Gamma$-algebra

$$
k(P)=\left\langle X ; \Gamma \mid u_{i}-v_{j}=0, j \in J\right\rangle=\langle X ; \Gamma \mid S\rangle
$$

(again we identify the relation $u-v=0$ with the pair $(u, v)$ ). Clearly, a relative GröbnerShirshov basis of the semigroup algebra (16) does not depend on $k$ and is simply a set of semigroup relations, say $S^{\text {comp }}$. This set $S^{\text {comp }}$ is called a relative Gröbner-Shirshov basis of the semigroup (15).

The $\Gamma$-free group $\operatorname{gr}\langle X ; \Gamma\rangle$ is defined as the group generated by $X$ and $\Gamma$ with the defining relations (1) and (3). In fact, this is the free $\Gamma$-semigroup generated by $X \cup X^{-1}$, with defining relations

$$
x_{i} x_{i}^{-1}=1 \quad \text { and } \quad x_{i}^{-1} x_{i}=1, \text { where } x_{i} \in X .
$$

Now, we can easily define the $\Gamma$-group

$$
G=\operatorname{gr}\langle X ; \Gamma \mid S\rangle
$$


generated by $X$ with defining relations $S$. The group algebra $k(G)$ has the following presentation:

$$
k(G)=\left\langle X \cup X^{-1} ; \Gamma \mid x_{i} x_{i}^{-1}=1, x_{i}^{-1} x_{i}=1, S\right\rangle .
$$

A relative Gröbner-Shirshov basis of the algebra (17) will be called a relative GröbnerShirshov basis of $G$ with respect to the set of generators $X$ and the order of the group of $X$-words.

\section{§3. GRoups LIKE $G\left(\overline{G F(2)\left(Q_{0}\right)}\right), Q_{0}=\operatorname{sgr}\langle w, h, u, f \mid w h=u f\rangle$}

We denote by $G F(2)\left(Q_{0}\right)$ the semigroup algebra of $Q_{0}$ over $G F(2)$. Then $\overline{G F(2)\left(Q_{0}\right)}$ is the completion of $G F(2)\left(Q_{0}\right)$ up to the algebra of formal infinite power series. It is a domain (see [23]). Let $\overline{G F(2)\left(Q_{0}\right)}$ * denote the multiplicative semigroup of nonzero elements. In [5], a certain representation of this semigroup was found in terms of generators and relations; we fix this presentation of ${\overline{G F(2)\left(Q_{0}\right)}}^{*}$. We use $G_{0}=G\left({\overline{G F(2)\left(Q_{0}\right)}}^{*}\right)$ to denote the universal group of fractions of the semigroup in question (recall that this is the group with the same generators and defining relations as $\overline{G F(2)\left(Q_{0}\right)}{ }^{*}$. An abstract definition of groups "like" $G_{0}$ was given in 14. Explicit examples of such groups can be found in [8]. These are groups of the form $G\left(\overline{G F(2)(Q)}^{*}\right)$, where $Q$ is the so-called SNA-semigroup with defining relations $w_{i} h_{i}=u_{i} f_{i}$, where $w_{i}, h_{i}, u_{i}, f_{i} \in X$, under some conditions. Here are some properties of these groups.

1) They are $\Gamma$-groups, where $\Gamma$ is the group of invertible series (units) of $\overline{G F(2)(Q)}$. For any $p$ in the fixed system of generators of the semigroup $\overline{G F(2)(Q)}$, we have

$$
\Gamma_{p}=\{1+p A\}, \quad \Gamma_{p}^{\prime}=\{1+A p\},
$$

where $A \in \overline{G F(2)(Q)}$.

2) In [8, for any $G=G\left(\overline{G F(2)(Q)}^{*}\right)$, where $Q$ is an SNA-semigroup, we constructed a rewriting system (semi-Thue-system)

$$
\sqcap=\left[\Sigma ; \Gamma \mid A_{j} \rightarrow B_{j}, j \in J\right]
$$

such that the set of canonical words (that contain no $A_{j}, j \in J$ ) is a $\Gamma$-basis of $G$ (i.e., any $\Gamma$-word is equal to a unique canonical $\Gamma$-word). Actually, for any $j \in J$ we have

$$
A_{j}>B_{j},
$$

in the "tower" order of the $\sum$-words.

Now we briefly describe the tower order of words. First, we let $X=Y \cup Z$ be a well-ordered set such that $z>y$ for any $z \in Z, y \in Y$. Suppose that the set $Y^{*}$ of $Y$ words can be well ordered in agreement with the order of all $Y$ and with concatenation of words. Any $X$-word has the form

$$
u=u_{0} z_{1} u_{1} \cdots u_{k} z_{k} u_{k+1}
$$

where $k \geq 0, u_{i} \in Y^{*}, s_{i} \in Z$. We define

$$
w t(u)=\left(k, u_{0}, z_{1}, u_{1}, \ldots, u_{k}, z_{k}, u_{k+1}\right)
$$

and order all the $w t$ 's lexicographically. Then the tower order of the $X$-words is defined as follows:

$$
u>v \Leftrightarrow w t(u)>w t(v) .
$$

Clearly, this is a well-ordering compatible with concatenation of words.

Property 2) above and Theorem 2.3 (the relative composition-diamond lemma) imply the following statement. 
Theorem 3.1. Let

$$
\sqcap=\left[\Sigma ; \Gamma \mid A_{i} \rightarrow B_{j}, j \in J\right]
$$

be the rewriting system for $G=G\left(\overline{G F(2)(Q)}^{*}\right)$, where $Q$ is an $S N A$-semigroup [8. Then the set

$$
S=\left\{A_{j}-B_{j}, j \in J\right\} \subset G F(2)\langle\Sigma ; \Gamma\rangle
$$

is the relative $\Gamma$-Gröbner-Shirshov basis of $G$ with respect to the tower order of words.

Corollary 3.2 ([8]). Any semigroup $\overline{G F(2)(Q)}{ }^{*}$, where $Q$ is an SNA-semigroup, is embeddable in a group.

Now, we return to $S N A$-semigroups $Q$ [8. A crucial property of $Q$ is that $Q$ has no left (right) cycles of order not exceeding 3 in the sense of Adyan [1]; in particular, $Q$ admits no relations of the form

$$
w h=u f, \quad w_{1} h_{1}=u f_{1}, \quad w_{1} h_{2}=w f_{2} .
$$

On the contrary, the semigroup $Q_{4}$ (see $[8,9]$ ) is defined by the same relations as in the Introduction:

$$
a_{i} s_{i}=c_{i} v_{0}, \quad b_{i} s_{i+1}=c_{i} v_{1}, \quad b_{i} t_{i+1}=a_{i} t_{i},
$$

where $1 \leq i \leq 4, s_{5}=s_{1}, t_{5}=t_{1}$. This system of relations is similar to (18). Consequently, $Q_{4}$ is not an $S N A$-semigroup.

Here we note that the algebra $G F(2)\left(Q_{4}\right)$ is not embeddable into a division algebra; it is not even invertible. (A domain $D$ is said to be invertible if $D \subset D_{1}$, where $D_{1}$ is a domain such that any nonzero element of $D$ is invertible in $D_{1}$ ). Indeed, suppose that $D=G F(2)\left(Q_{4}\right)$ is contained in $D_{1}$ with the above property. Then in $D_{1}$ we have

$$
v_{0}^{-1} v_{1}=s_{i}^{-1} a_{i}^{-1} b_{i} s_{i+1}=s_{i}^{-1} t_{i} t_{i+1}^{-1} s_{i+1}, \quad 1 \leq i \leq 4,
$$

whence

This means that

$$
\left(v_{0}^{-1} v_{1}\right)^{4}=1
$$

$$
\left(v_{0}^{-1} v_{1}-1\right)^{4}=0
$$

in $D_{1}$, and $v_{0}^{-1} v_{1}=1$, so that $v_{0}=v_{1}$ in $D$, a contradiction.

Remark 3.3. The above argument shows that the algebra $G F(2)\left(Q_{4}\right)$ cannot be embedded in any domain $D_{1}$ with $Q_{4} \subset U\left(D_{1}\right)$, where $U\left(D_{1}\right)$ is the group of units of $D_{1}$.

Let $\overline{G F(2)\left(Q_{4}\right)}$ be the completion of $G F(2)\left(Q_{4}\right)$. Then the main results of [6, [8, 9 ] take the following form.

Main Theorem. The semigroup $\overline{G F(2)\left(Q_{4}\right)}$ ' is embeddable in a group.

For the proof of this theorem, the concept of an "almost" $\Gamma$-normal form was constructed for the group $G_{4}=G\left(\overline{G F(2)\left(Q_{4}\right)}\right)^{*}$. Here, as before, $\Gamma$ is the group of units of $\overline{G F(2)\left(Q_{4}\right)}$, and

$$
\Gamma_{q}=\{1+q A\}, \quad \Gamma_{q}^{\prime}=\left\{1+A_{q}\right\},
$$

where $q$ is among the fixed generators of $\overline{G F(2)\left(Q_{4}\right)}{ }^{*}$, and $A \in \overline{G F(2)\left(Q_{4}\right)}$. The only problem ("almost") is that we changed the generator $v_{1}$ of $G$ to

$$
p=v_{0}^{-1} v_{1} .
$$

But all arguments of [8] and [9] are still valid for $v_{1}$ in place of $p$. This can be checked directly. We only need to change the presentation of the first $\Gamma$-group $G_{1}$ in 9 ]:

$$
G_{1}=\left\langle v_{0} ; v_{1} ; \Gamma \mid\left(v_{0}^{-1} v_{1}\right)^{4}=1\right\rangle .
$$


Thus, the relative Gröbner-Shirshov basis of this new $\Gamma$-group $G_{1}$ consists only of trivial relations and the relations

$$
\begin{aligned}
& v_{0}^{-1} v_{1} v_{0}^{-1} v_{1}=v_{1}^{-1} v_{0} v_{1}^{-1} v_{0}, \\
& v_{0} v_{1}^{-1} v_{0} v_{1}^{-1}=v_{1} v_{0}^{-1} v_{1} v_{0}^{-1},
\end{aligned}
$$

where the left-hand sides are maximal words in the tower order relative to

$$
\left\{v_{0}^{-1}, v_{0}\right\} \dot{\cup}\left\{v_{1}^{-1}, v_{1}\right\},
$$

and $v_{0}^{-1}<v_{0}<v_{1}^{-1}<v_{1}$.

So, the results of 8 and 9 lead to the following statement.

Theorem 3.4. Let

$$
\sqcap_{4}=\left[\Sigma ; \Gamma \mid A_{j} \rightarrow B_{j}, j \in J\right]
$$

be the rewriting (semi-Thue) system constructed for $G\left(\overline{G F(2)\left(Q_{4}\right)}{ }^{*}\right)$ in [9], with (20) in place of the transformations $p^{4} \rightarrow 1, p^{-1} \rightarrow p^{3}$. Then the relations

$$
S_{4}=\left\{A_{j}-B_{j}, j \in J\right\}
$$

constitute a relative $(\Gamma)$-Gröbner-Shirshov basis for the group $G\left({\overline{G F(2)\left(Q_{4}\right)}}^{*}\right)$ with respect to the tower order of group words.

The main theorem mentioned above follows immediately from Theorem 3.4. On the other hand, we could use the relative composition-diamond lemma (Theorem 2.3) to prove Theorem 3.4 by checking that all possible compositions of the polynomials (21) are trivial. In principle, this approach would be easier than the rather complicated arguments used in [8] and [9 based on the notion of a "group with a relative standard basis" [7].

\section{§4. Digression to SOME OPEn PROBlEMs FOR SEMigroup AND GROUP ALGEBRAS}

The following are some classes of domains (i.e., associative rings without divisors of zero, see 13 ):

$\mathcal{D}_{0}$ - all domains;

$\mathcal{D}_{1}$ - all domains with multiplicative semigroups embeddable into groups;

$\mathcal{D}_{2}$ - all invertible domains (see $\S 3$ );

$\mathcal{D}_{3}$ - all domains embeddable into fields; then

$$
\mathcal{D}_{0} \supseteq \mathcal{D}_{1} \supseteq \mathcal{D}_{2} \supseteq \mathcal{D}_{3} .
$$

Malcev's famous example 23] shows that $\mathcal{D}_{0} \neq \mathcal{D}_{1}$. In particular, it follows that $\mathcal{D}_{0} \neq \mathcal{D}_{3}$, which solves the problem of Van der Waerden (see [26]).

The example given by Bokut in [6, 8, 9] shows that $\mathcal{D}_{1} \neq \mathcal{D}_{2}$. The examples given by Bowtell [3] and Klein [20, together with the result of Gerasimov in [18], show that $\mathcal{D}_{2} \neq \mathcal{D}_{3}$ (see also [17]). In fact, these three examples give a complete solution to the Malcev problem.

Remark 4.1. A. I. Malcev formulated his problem $\left(\mathcal{D}_{1} \neq \mathcal{D}_{3}\right)$ around 1937 (see 24, p. 5]). At the Moscow ICM, 1966, the solution of the Malcev problem was announced by L. A. Bokut, by P. M. Cohn on behalf of his student A. Bowtell, and by S. Amitsur on behalf of his student A. A. Klein. Next year, three papers on this subject were published (see [6, 3, 20, ). The paper [6] was submitted by A. I. Malcev to the Russian Academy of Sciences Doklady. A full proof of Bokut's example appeared in [8, 9], as has already been mentioned. 
In fact, the Malcev and Bokut examples are semigroup algebras. Note that, up to now, the Bokut example is the only known example of a semigroup algebra in $\mathcal{D}_{1} \backslash \mathcal{D}_{2}$.

To present the situation for semigroup algebras, we let $\mathcal{S}_{i}$ be the intersection of $\mathcal{D}_{i}$ with the class of semigroup algebras, $0 \leq i \leq 3$. Then

$$
\mathcal{S}_{0} \supset \mathcal{S}_{1} \supset \mathcal{S}_{2} \supseteq \mathcal{S}_{3}
$$

However, so far there are no examples of semigroup algebras in $\mathcal{D}_{2} \backslash \mathcal{D}_{3}$.

Very interesting open problems arise if the domains in (23) are group algebras over a field. Consider the following classes of algebras:

$\mathcal{K}$ - all group algebras of torsion free groups;

$\mathcal{K}_{0}$ - all group algebras without zero divisors (i.e., domains);

$\mathcal{K}_{1}$ - all group algebras that are domains with multiplicative semigroups embeddable into groups;

$\mathcal{K}_{2}$ - all invertible group algebras;

$\mathcal{K}_{3}$ - all group algebras embeddable into fields.

Then

$$
\mathcal{K} \supseteq \mathcal{K}_{0} \supseteq \mathcal{K}_{1} \supseteq \mathcal{K}_{2} \supseteq \mathcal{K}_{3}
$$

The question as to whether $\mathcal{K} \neq \mathcal{K}_{0}$ becomes the famous Kaplansky zero-divisor problem [19.

The question as to whether $\mathcal{K}_{0} \neq \mathcal{K}_{3}$ is a problem similar to the Van der Waerden problem in the class of group algebras.

The question as to whether $\mathcal{K}_{1} \neq \mathcal{K}_{3}$ is a problem similar to the Malcev problem in the class of group algebras.

So far, all the proper embedding questions in (24) remain open and difficult problems.

\section{$\S 5$. TITS SYSTEMS}

In this section, we shall show that the well-known Bruhat decompositions of elements of a group $G$ involved in a Tits system $(G, B, N, S)$ are Irr-words with respect to a relative (B)-Gröbner-Shirshov basis of $G$ (see, e.g., 2]). The definition of a Tits system $(G, B, N, S)$ is as follows. First, let $G$ be a group, and let $B$ and $N$ be subgroups of $G$. Suppose $T=B \cap N$ is a normal subgroup of $N$ and $S \subset W=N / B \cap N$.

Then, we present $B, N$, and $T$ by the following multiplication tables:

$$
\begin{aligned}
& B=\left\langle\{b\} \mid b b^{\prime}=b^{\prime \prime}\right\rangle, \\
& N=\left\langle\{n\} \mid n n^{\prime}=n^{\prime \prime}\right\rangle, \\
& T=\left\langle\{t\}=B \cap N \mid t t^{\prime}=t^{\prime \prime}\right\rangle .
\end{aligned}
$$

A system $(G, B, N, S)$ is called a Tits system if the following axioms are fulfilled (see [2]).

(T1) The set $B \cup N$ generates $G$, i.e.,

$$
(\forall g \in G) \quad g=b_{0} n_{1} \cdots b_{k-1} n_{k} b_{k},
$$

where $k \leq 0, b_{i} \in B, n_{i} \in N$.

(T2) $S$ generates $W$ and all elements of $S$ are of order 2 .

(T3) $(\forall s \in\{s\}), s B w \subset B w B \cup B s w B$.

(T4) $(\forall s \in\{s\}) \quad s B s \nsubseteq B$.

Since $\{w\}$ is a system of representatives of the cosets $n T, n \in N$, we have

$$
\begin{array}{ll}
(\forall n \in N) & n=w t, \\
\left(\forall w, w^{\prime} \in\{w\}\right) & w w^{\prime}=w^{\prime \prime} t, \\
(\forall t \in T, w \in\{w\}) & t w=w t^{\prime},
\end{array}
$$


where all $w$ 's belong to $\{w\}$ and all $t$ 's belong to $T$. The third identity in (27) shows that, for a fixed $w$, the map

$$
t \rightarrow t^{\prime}
$$

is an automorphism of $T$.

By (26) and (27), we have

$$
(\forall g \in G) \quad g=b_{0} w_{1} b_{1} \cdots w_{k} b_{k},
$$

where $k \geq 0, b_{i} \in B, w_{i} \in\{w\}$. Thus, $G$ is generated by $B$ and $\{w\}$.

Next, axiom (T2) implies that

$$
\begin{gathered}
(\forall w \in\{w\}) \quad w=s_{1} \cdots s_{n} t, \quad s_{i} \in S, \quad t \in T, \\
(\forall s \in\{s\}) \quad s^{2}=t, t \in T .
\end{gathered}
$$

Indeed,

$$
\begin{aligned}
w & =s_{1} t_{1} s_{2} t_{2} s_{3} t_{3} \cdots s_{n} t_{n} \\
& =s_{1} s_{2} t_{1}^{\prime} t_{2} s_{3} t_{3} \cdots s_{n} t_{n} \\
& =s_{1} s_{2} s_{3} t_{1}^{\prime \prime} t_{2}^{\prime} t_{3} \cdots s_{n} t_{n} \\
& =\cdots \\
& =s_{1} \cdots s_{n} t .
\end{aligned}
$$

Axiom (T3) shows that

$$
(\forall s \in\{s\}, b \in B, w \in\{w\}) \quad s b w=b_{1} s^{\delta} w b_{2},
$$

where $\delta=0,1 ; b_{1}, b_{2} \in B$.

Moreover,

$$
\left(\forall w_{1}, w_{2} \in\{w\}, b \in B\right) \quad w_{1} b w_{2}=b_{1} w b_{2},
$$

for some $b_{1}, b_{2} \in B, w \in\{w\}$. Indeed, by (29), $w_{1}$ is representable in the form $w_{1}=$ $s_{1}, \ldots, s_{n} t$; next, $b_{0}=t b \in B$, and (31) implies

$$
w_{1} b w_{2}=s_{1} \cdots s_{n} t b w_{2}=s_{1} \cdots s_{n} b_{0} w_{2}=b_{1} s_{1}^{\delta_{1}} \cdots s_{n}^{\delta_{n}} w_{2} b_{2}^{\prime}=b_{1} w t^{\prime} b_{2}^{\prime}=b_{1} w b_{2},
$$

where $\delta_{i}=0,1$.

Finally, (T4) means that

$$
(\forall s \in\{s\} \exists b \in B) \quad s b s \notin B .
$$

The above axioms (more precisely, T1-T3) imply that $G$ is generated by $B$ and $\{w\}$, with the defining relations (25), (27), and (31). For any $w \in\{w\}$, we define

$$
\begin{aligned}
& \Gamma_{w}=\left\{\gamma \in B \mid\left(\exists \gamma^{\prime} \in B\right) \gamma w=w \gamma^{\prime}\right\}, \\
& \Gamma_{w}^{\prime}=\left\{\gamma^{\prime} \in B \mid(\exists \gamma \in B) w \gamma^{\prime}=\gamma w\right\} .
\end{aligned}
$$

Then $\Gamma_{w}, \Gamma_{w}^{\prime}$ are two isomorphic subgroups of $B$ that contain $T$. Also, the isomorphism $\Gamma_{w} \rightarrow \Gamma_{w}^{\prime}, \gamma \mapsto \gamma^{\prime}$, extends the automorphism $T \rightarrow T, t \mapsto t^{\prime}$.

Now we see that $G$ is indeed a $B$-group with the following defining $B$-group relations:

$$
\begin{aligned}
w_{1} b w_{2} & =b_{1} w b_{2}, \\
w_{1} w_{2} & =w t .
\end{aligned}
$$

By using (35), we can show that any element $g$ of $G$ can be presented in the form $g=b_{1} w b_{2}$.

Clearly, this is the Bruhat decomposition of $g$. It is well known that a Bruhat decomposition is unique:

$$
b_{1} w b_{2}=b_{1}^{\prime} w^{\prime} b_{2}^{\prime} \Rightarrow w=w^{\prime}
$$


(see, e.g., 2]). Then we have

$$
\gamma=b_{1}^{\prime-1} b_{1} \in \Gamma_{w}, \quad \gamma^{\prime}=b_{2}^{\prime} b_{2}^{-1} \in \Gamma_{w}^{\prime} .
$$

This shows the uniqueness of the Bruhat decomposition (36) in the sense of $B$-words.

Now, using Theorem 2.3 (the relative composition-diamond lemma) and assuming that $\{w\}$ is well ordered and the set of $B$-words on $\{w\}$ is ordered in the deg-lex order, we obtain the following result.

Theorem 5.1. Let $(G, B, N, S)$ be a Tits system. The relations

$$
\begin{aligned}
w_{1} b w_{2} & =b_{1} w b_{2}, \\
w_{1} w_{2} & =w t,
\end{aligned}
$$

where $w, w_{i} \in\{w\}, b, b_{i} \in B, t \in T$, form a relative $(B)$-Gröbner-Shirshov basis of $G$ as a $B$-group in the generators $\{w\}$. Next, the Irr-words of $G$ with respect to the above relative Gröbner-Shirshov basis are the Bruhat decompositions (words) of G.

We illustrate Theorem 5.1 by $G=S L_{2}(k)$. We follow the notation of [21].

Suppose $k$ is a field, $b \in k, a \in k \backslash\{0\}$. Write

$$
u(b)=\left(\begin{array}{ll}
1 & b \\
0 & 1
\end{array}\right), \quad t(a)=\left(\begin{array}{cc}
a & 0 \\
0 & a^{-1}
\end{array}\right), \quad \text { and } \quad w=s=\left(\begin{array}{cc}
0 & 1 \\
-1 & 0
\end{array}\right) .
$$

Then $T=\{t(a), a \in k \backslash\{0\}\}$ is a maximal torus subgroup of $G, U=\{u(b), b \in k\}$ is a unipotent subgroup of $G$,

$$
B=U T=\left\{\left(\begin{array}{cc}
a & b \\
0 & a^{-1}
\end{array}\right) \in k \backslash\{0\}, b \in k\right\}
$$

is a Borel subgroup of $G$, and

$$
N=T \cup w T=\left\{\left(\begin{array}{cc}
a & 0 \\
0 & a^{-1}
\end{array}\right),\left(\begin{array}{cc}
0 & a^{-1} \\
a & 0
\end{array}\right), a \in k \backslash\{0\}\right\} .
$$

Clearly, $N$ is a subgroup of $G$ and $N \cap B=T \triangleleft N$ is a normal subgroup of $N$. Then $(G, B, N, S=\{w T\})$ is a Tits system and the following relations are valid:

$$
\begin{aligned}
w u(a) w & =u(a) w t(-a) u(a), \\
w^{2} & =t(-1), \\
u\left(b_{1}\right) u\left(b_{2}\right) & =u\left(b_{1}+b_{2}\right), \\
t\left(a_{1}\right) t\left(a_{2}\right) & =t\left(a_{1} a_{2}\right), \\
t(a) u(b) & =u\left(b a^{2}\right) t(a), \\
t(a) w & =w t(a) .
\end{aligned}
$$

Moreover,

$$
s B s=\left\{\left(\begin{array}{cc}
a^{-1} & 0 \\
b & a
\end{array}\right)\right\} \not \subset B .
$$

In this case $\Gamma_{w}=\Gamma_{w}^{\prime}=\Gamma$. System (37) is a $B$-relative Gröbner-Shirshov basis of $G$ in the deg order of $B$-words in $\{w\}$. Moreover, in this case, (37) may be regarded as an (absolute) Gröbner-Shirshov basis if $U$ is well ordered, $T$ is ordered arbitrarily, and then the words in $U \cup T$ are ordered by using deg-lex order, assuming $u(b)>t(a)$. Then, we order the words in $(U \cup T) \cup\{w\}$ in the tower order. Now, by direct computation of compositions, it is easy to show that (37) is complete under compositions not only as a $B$-set, but in the absolute sense.

Using the usual composition-diamond lemma $(\Gamma=1)$, we obtain the following statement. 
Theorem 5.2. Any element of $S L_{2}(k)$ has a unique presentation in the form

$$
u(b) t(a) \text { or } u(b) w u(c) t(a) .
$$

Results such as Theorems 5.1 and 5.2 can be checked not only for $S L_{2}(k)$, but also for any Chevalley group (see [16, Theorem 8.4.3]), by applying the composition-diamond lemma with $\Gamma=1$.

\section{REFERENCES}

[1] S. I. Adyan, Defining relations and algorithmic problems for groups and semigroups, Trudy Mat. Inst. Steklov. 85 (1966), 123 pp.; English transl., Proc. Steklov Inst. Math. No. 85 (1966), 152 pp. MR 0204501(34:4340) MR0218434(36:1520)

[2] N. Bourbaki, Lie groups and Lie algebras, Chapters 4-6, Elements of Mathematics, Springer-Verlag, Berlin, 2002. MR 1890629 (2003a:17001)

[3] A. Bowtell, On a question of Mal'cev, J. Algebra 7 (1967), 126-139. MR0230750 (37:6310)

[4] L. A. Bokut', Some examples of rings without zero divisors, Algebra i Logika Sem. 3 (1964), no. 5-6, 5-28. (Russian) MR0174586 (30:4787)

[5] - Factorization theorems for certain classes of rings without divisors of zero. I, II, Algebra i Logika Sem. 4 (1965), no. 4, 25-52; 4 (1965), no. 5, 17-46. (Russian) MR0197501 (33:5666) MR0197502 (33:5667)

[6] The imbedding of rings in fields, Dokl. Akad. Nauk SSSR 175 (1967), no. 4, 755-758; English transl., Soviet Math. Dokl. 8 (1967), 901-904. MR0222115 (36:5167)

[7] _ Groups with a relative standard basis, Sibirsk. Mat. Zh. 9 (1968), no. 3, 499-521; English transl., Siberian Math. J. 9 (1968), 377-393. MR0237671 (38:5952)

[8] _ Groups of fractions of multiplicative semigroups of certain rings. I, II, III, Sibirsk. Mat. Zh. 10 (1969), no. 2, 246-286; ibid. 10 (1969), no. 4, 744-799; ibid. 10 (1969), no. 4, 800-819; English transl., Siberian Math. J. 10 (1969), 172-203; ibid. 10 (1969), 541-582; ibid. 10 (1969), 583-600. MR0255606 (41:267a)

[9] _ The problem of Mal'cev, Sibirsk. Mat. Zh. 10 (1969), no. 5, 965-1005; English transl., Siberian Math. J. 10 (1969), 706-739. MR0255607(41:267b)

[10] Unsolvability of the word problem, and subalgebras of finitely presented Lie algebras, Izv. Akad. Nauk SSSR Ser. Mat. 36 (1972), 1173-1219; English transl., Math. USSR-Izv. 6 (1972), 1153-1199 (1973). MR0330250(48:8588)

[11] _ Imbeddings into simple associative algebras, Algebra i Logika 15 (1976), no. 2, 117-142. (Russian) MR 0506423(58:22167)

[12] Malcev's problem and groups with a normal form (With the collaboration of D. J. Collins), Stud. Logic Found. Math., vol. 95, Word Problems, II (Conf. on Decision Problems in Algebra, Oxford, 1976), North-Holland, Amsterdam-New York, 1980, pp. 29-53. MR0579936 (82c:20064)

[13] _ Imbedding of rings, Uspekhi Mat. Nauk 42 (1987), no. 4, 87-111; English transl., Russian Math. Surveys 42 (1987), no. 4, 105-138. MR.0912062 (88m:16003)

[14] - Abstract semigroups and groups of formal series of dependent variables, Siberian Adv. Math. 6 (1996), no. 3, 1-26. MR1469036 (99c:20093)

[15] L. A. Bokut' and P. S. Kolesnikov, Gröbner-Shirshov bases: From inception to the present time, Zap. Nauchn. Sem. S.-Peterburg Otdel. Mat. Inst. Steklov. (POMI) 272 (2000), 26-67; English transl., J. Math. Sci. (N. Y.) 116 (2003), no. 1, 2894-2916. MR.1811792 (2002a:16030)

[16] R. Carter, Simple groups of Lie type, Wiley Classical Library, A Wiley-Interscience Publ., John Wiley and Sons., Inc., New York, 1989. MR1013112 (90g:20001)

[17] P. M. Cohn, Free rings and their relations, 2nd ed., London Math. Soc. Monogr., vol. 19, Acad. Press, Inc., London, 1985. MR0800091 (87e:16006)

[18] V. N. Gerasimov, Inverting homomorphisms of rings, Algebra i Logika 18 (1979), no. 6, 648-663; English transl., Algebra and Logic 18 (1979), no. 6, 408-420 (1980). MR0596422 (82d:16003)

[19] I. Kaplansky, Problems in the theory of rings, Report of a Conference on Linear Algebras, June, 1956, National Academy of Sciences-National Research Council, Washington, Publ. 502, 1957, pp. 1-3. MR0096696(20:3179)

[20] A. A. Klein, Rings nonembeddable in fields with multiplicative semi-groups embeddable in groups, J. Algebra 7 (1967), 100-125. MR0230749 (37:6309)

[21] S. Lang, $S L_{2}(R)$, Grad. Texts in Math., vol. 105, Springer-Verlag, New York, 1985. MR0803508 (86j:22018) 
[22] D. Lazard, Gröbner bases, Gaussian elimination and resolution of systems of algebraic equations, Computer Algebra (London, 1983), Lecture Notes in Comput. Sci., vol. 162, Springer, Berlin, 1983, pp. 146-156. MR0774807 (86m:13002)

[23] A. I. Malcev, On the immersion of an algebraic ring into a field, Math. Ann. 113 (1937), 686-691. MR 1513116

[24] , Selected works. Vol. 1. Classical algebra, "Nauka", Moscow, 1976. (Russian) MR0424502 $(54: 12464)$

[25] A. I. Shirshov, Some algorithm problems for Lie algebras, Sibirsk. Mat. Zh. 3 (1962), no. 2, 292-296; English transl., ACM SIGSAM Bull. 33 (1999), no. 2, 3-6. MR0183753 (32:1231)

[26] B. L. van der Waerden, Modern algebra. Vol. 1, Frederick Ungar Publ. Co., New York, NY, 1949. MR.0029363 (10:587b)

Sobolev Institute of Mathematics, Russian Academy of Sciences, Siberian Branch, NovosiBIRSK, 630090, RUSSIA

E-mail address: bokut@math.nsc.ru

Department of Mathematics, the University of Hong Kong, Pokfulam Road, Hong Kong, CHINA (SAR)

E-mail address: kpshum@maths.hku.hk

Received 6/AUG/2007

Translated by THE AUTHORS 